\title{
Isospin Breaking in Heavy-Meson Decay Constants
}

\author{
Wolfgang Lucha \\ Institute for High Energy Physics, Austrian Academy of Sciences, Nikolsdorfergasse 18, A-1050 \\ Vienna, Austria \\ E-mail: Wolfgang.Lucha@oeaw.ac.at
}

\section{Dmitri Melikhov*}

Institute for High Energy Physics, Austrian Academy of Sciences, Nikolsdorfergasse 18, A-1050

Vienna, Austria, and

D. V. Skobeltsyn Institute of Nuclear Physics, M. V. Lomonosov Moscow State University,

119991, Moscow, Russia, and

Faculty of Physics, University of Vienna, Boltzmanngasse 5, A-1090 Vienna, Austria

E-mail: dmitri_melikhov@gmx.de

\section{Silvano Simula}

INFN, Sezione di Roma Tre, Via della Vasca Navale 84, I-00146 Roma, Italy

E-mail: simula@roma3.infn.it

Evaluation of Borelized QCD sum rules in the so-called local-duality limit of infinitely large Borel mass parameter provides an alternate route for extraction of the dependence of the decay constants of heavy-light mesons on the mass $m_{q}$ of the involved light quark $q$ : For appropriate choices of the two-point correlation functions of currents interpolating the hadrons under study, the local-duality limit forces all nonperturbative contributions parametrized by vacuum condensates to such kind of correlator to vanish. As a consequence, the sought $m_{q}$ dependence of the heavy-light meson decay constants proves to be controlled primarily by the correlator contributions from perturbative QCD. Our knowledge of the analytic behaviour of the latter as functions of $m_{q}$ enables us to derive the $m_{q}$ dependence of the decay constants of both pseudoscalar and vector heavy-light mesons, for which we estimate strong isospin breaking to be of the order of $1 \mathrm{MeV}$ for both charm and beauty sectors.

XVII International Conference on Hadron Spectroscopy and Structure - Hadron2017

25-29 September, 2017

University of Salamanca, Salamanca, Spain

\footnotetext{
${ }^{*}$ Speaker.
} 


\section{Local-Duality Limit of QCD Sum Rules for Heavy-Light Meson Decay Constants}

QCD sum rules [1] constitute a nonperturbative approach to bound states of quarks and gluons, the degrees of freedom of quantum chromodynamics (QCD), the quantum field theory governing all strong interactions. This kind of relations may be constructed by evaluating, at the level of QCD and at the hadronic level, correlation functions of operators appropriately defined in terms of quarks and gluons but interpolating the hadron of interest. Application of Borel transformations from momenta to new variables, the Borel parameters $\tau$, suppresses (unwanted) hadronic-continuum contributions. For heavy-light mesons $H_{q}$ of mass $M_{H_{q}}$ composed of a heavy quark $Q=c, b$ of mass $m_{Q}$ and a light quark $q=u, d, s$ of mass $m_{q}$, the Borel QCD sum rules for their decay constants $f_{H_{q}}$ generically read

$$
f_{H_{q}}^{2}\left(M_{H_{q}}^{2}\right)^{N} \exp \left(-M_{H_{q}}^{2} \tau\right)=\int_{\left(m_{Q}+m_{q}\right)^{2}}^{s_{\text {eff }}^{(N)}\left(\tau, m_{Q}, m_{q}, \alpha_{\mathrm{s}}\right)} \mathrm{d} s \exp (-s \tau) s^{N} \rho\left(s, m_{Q}, m_{q}, \alpha_{\mathrm{s}}\right)+\Pi^{(N)}\left(\tau, m_{Q}, m_{q}, \alpha_{\mathrm{s}},\langle\bar{q} q\rangle, \ldots\right),
$$

with a positive integer exponent $N=0,1, \ldots$ fixed by the detailed formulation of the QCD sum rule. The spectral densities $\rho\left(s, m_{Q}, m_{q}, \alpha_{\mathrm{s}}\right)$ can be found in form of expansions in the strong coupling $\alpha_{\mathrm{s}}$. The $\tau$-dependent effective threshold $s_{\text {eff }}^{(N)}\left(\tau, m_{Q}, m_{q}, \alpha_{\mathrm{s}}\right)$ forms the lower boundary of that region of $s$ (extending to infinity) over which, by the postulate of quark-hadron duality, mutual cancellations of the contributions of perturbative QCD and of hadronic excitations and continuum should take place. Basically, nonperturbative effects manifest in QCD sum rules in two places: as vacuum condensates in power corrections $\Pi^{(N)}\left(\tau, m_{Q}, m_{q}, \alpha_{\mathrm{s}},\langle\bar{q} q\rangle, \ldots\right)$, power series in $\tau$, and in $s_{\text {eff }}^{(N)}$. Depending on that (in fact, chosen) number $N$, their relative fractions in power corrections and effective threshold vary.

The conventional procedure of deriving, from such QCD sum rule, the sought relation between the hadron characteristics of interest and the fundamental parameters of QCD starts by identifying a suitable interval of (thus inevitably almost everywhere nonzero) values of $\tau$, defined such that, at the hadron side, the ground-state contribution is reasonably large and, at the QCD side, nonperturbative corrections stay sufficiently small. Equipped with the increase of the accuracy [2] of the predictions gained by taking seriously the $\tau$ dependence [3] of the effective threshold, and determining the latter by minimizing the discrepancy between theoretical hadron masses and their true values known from experiment, we managed to extract precise decay-constant predictions [4,5] from the required set of QCD quantities, such as quark masses, strong coupling, spectral densities and vacuum condensates.

The - compared to typical hadron masses tiny - difference $\left(m_{d}-m_{u}\right)(2 \mathrm{GeV}) \approx 2.5 \mathrm{MeV}$ [6] of the down-quark mass $m_{d}$ and the up-quark mass $m_{u}$ generates strong-isospin breakdown reflected by the difference $f_{H_{d}}-f_{H_{u}}$ of the decay constants of heavy-light mesons $H_{d}$ and $H_{u}$ involving, apart from a given heavy quark, a light $d$ and $u$ quark, respectively. Our QCD sum-rule version relying on $\tau$-dependent thresholds proves to be a tool so sharp that we can reliably treat such phenomena $[7,8]$.

We may look at this manifestation of isospin breaking from a related but slightly different angle [9], namely, by applying the QCD sum-rule formalism sketched above in the so-called local-duality limit, ${ }^{1}$ realized by the Borel variable approaching its lower boundary, that is, by $\tau \rightarrow 0$, to Borelized correlation functions of mass dimension two, corresponding to exponent $N=0$. In this well-defined limit, the power corrections $\Pi^{(N)}$ vanish, the QCD sides of the emerging QCD sum rules simplify to dispersion integrals of the spectral densities $\rho\left(s, m_{Q}, m_{q}, \alpha_{\mathrm{s}} \mid m_{\text {sea }}\right)$ (indicating also their dependence

\footnotetext{
${ }^{1}$ This limit of QCD sum rules has been applied to pion and nucleon elastic and meson transition form factors [10,11].
} 
on the masses $m_{\text {sea }}$ of all sea quarks showing up in higher-order corrections), and all nonperturbative effects are incorporated by their upper limits of integration, the effective thresholds $s_{\mathrm{eff}}\left(m_{Q}, m_{q}, \alpha_{\mathrm{s}}\right)$ :

$$
f_{H_{q}}^{2}=\int_{\left(m_{Q}+m_{q}\right)^{2}}^{s_{\mathrm{eff}}\left(m_{Q}, m_{q}, \alpha_{\mathrm{s}}\right)} \mathrm{d} s \rho\left(s, m_{Q}, m_{q}, \alpha_{\mathrm{s}} \mid m_{\text {sea }}\right) \equiv \digamma\left(s_{\mathrm{eff}}\left(m_{q}, \cdot\right), m_{q} \mid m_{\text {sea }}\right) .
$$

For simplicity of notation, we denote the QCD side by $\digamma\left(s_{\text {eff }}\left(m_{q}, \cdot\right), m_{q} \mid m_{\text {sea }}\right)$ and highlight only its dependence on the light-quark masses. At the hadron side, in the local-duality limit any dependence on the mass of the ground state disappears, whose footprint is reduced to its decay constant squared. In the spectral densities' perturbative expansions (Fig. 1), sea quarks begin to contribute at order $\alpha_{\mathrm{s}}^{2}$ : $\rho\left(s, m_{Q}, m_{q}, \alpha_{\mathrm{s}} \mid m_{\text {sea }}\right)=\rho_{0}\left(s, m_{Q}, m_{q}\right)+\frac{\alpha_{\mathrm{s}}(\mu)}{\pi} \rho_{1}\left(s, m_{Q}, m_{q}, \mu\right)+\frac{\alpha_{\mathrm{s}}^{2}(\mu)}{\pi^{2}} \rho_{2}\left(s, m_{Q}, m_{q}, \mu \mid m_{\text {sea }}\right)+\cdots$. Truncations of such perturbative series lead to unphysical dependences on renormalization scales $\mu$.

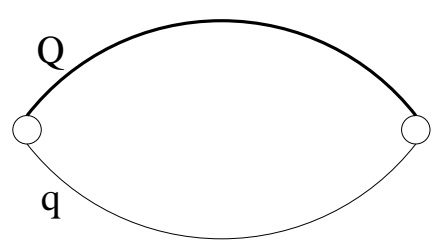

(a)

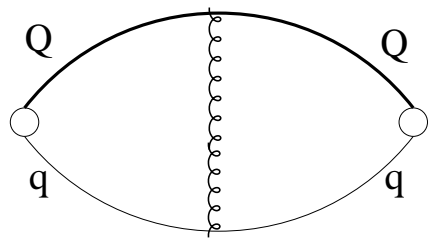

(b)

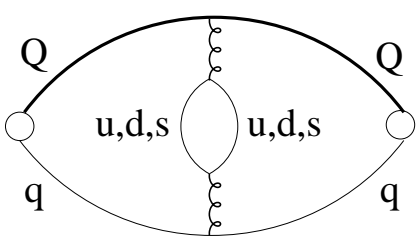

(c)

Figure 1: Two-point $(Q \bar{q})$ correlator: leading (a), next-to-leading (b), next-to-next-to-leading (c) order in $\alpha_{\mathrm{s}}$.

The heavy-light spectral densities required as input have been computed up to order $\alpha_{\mathrm{s}}^{2}$ [12]; at order $\alpha_{\mathrm{s}}^{2}$, however, only for the case of massless light quarks: $\rho_{2}\left(s, m_{Q}, m_{q} \mid m_{\text {sea }}\right) \approx \rho_{2}\left(s, m_{Q}, 0 \mid 0\right)$.

It is easy to convince oneself that adopting this local-duality limit $\tau \rightarrow 0$ is both mathematically well-defined and physically well-grounded: Traditional Borel QCD sum rules identify constants $s_{\text {eff }}$ such that within chosen Borel windows the predicted hadron observables exhibit the least sensitivity to the value of $\tau$. However, Fig. 2 illustrates that these regions can safely be extended down to $\tau=0$; the effective thresholds found by requiring Borel stability guarantee the latter to hold also for $\tau \rightarrow 0$.
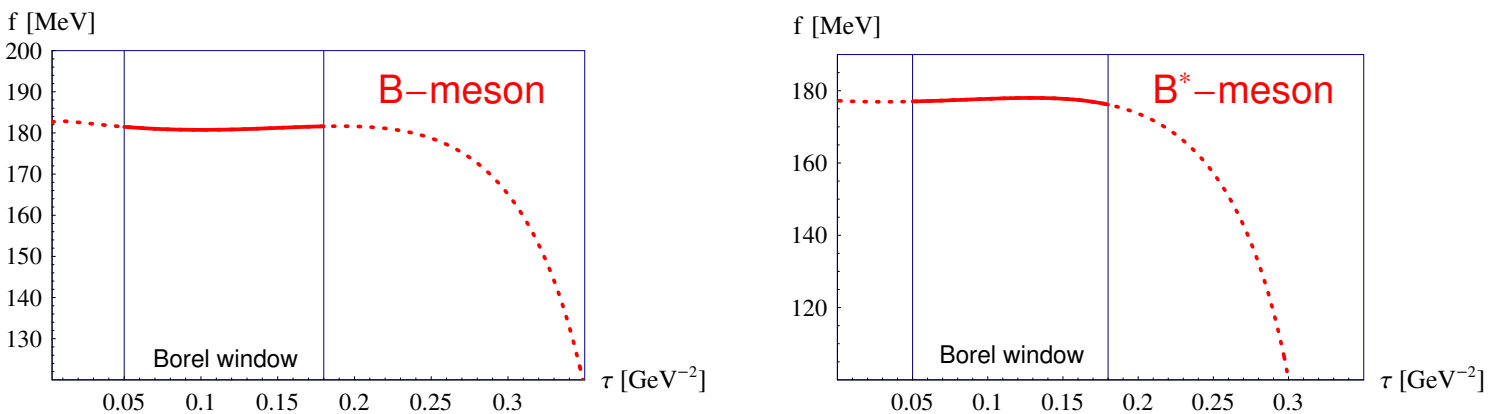

Figure 2: Predictions of Borel QCD sum rules for the $B$ - and $B^{*}$-meson decay constants [9] vs. Borel variable $\tau$, for one and the same effective threshold $s_{\text {eff }}$ within (solid line) and beyond (dotted line) the Borel windows. For both pseudoscalar and vector mesons, demanding Borel stability over the windows fixes the values of $s_{\mathrm{eff}}$. 


\section{Leptonic Decay Constant of Heavy-Light Meson: Light-Quark Mass Dependence}

We study the leptonic decay constants, $f_{P_{q}}$ and $f_{V_{q}}$, of pseudoscalar $\left(P_{q}\right)$ and vector $\left(V_{q}\right)$ mesons with masses $M_{P_{q}}$ and $M_{V_{q}}$, four-momentum $p$ and $V_{q}$ polarization vector $\varepsilon_{\mu}(p)$, defined according to

$$
\left\langle 0\left|\bar{q}(0) \gamma_{\mu} \gamma_{5} Q(0)\right| P_{q}(p)\right\rangle=\mathrm{i} f_{P_{q}} p_{\mu}, \quad\left\langle 0\left|\bar{q}(0) \gamma_{\mu} Q(0)\right| V_{q}(p)\right\rangle=f_{V_{q}} M_{V_{q}} \varepsilon_{\mu}(p) .
$$

The decay-constant discrepancy in the focus of our interest, $f_{H_{d}}-f_{H_{u}}$, betraying isospin breaking, is proportional to the difference of the spectral integral $\digamma\left(s_{\mathrm{eff}}\left(m_{q}, \cdot\right), m_{q} \mid m_{\text {sea }}\right)$ in Eq. (1.1) if evaluated
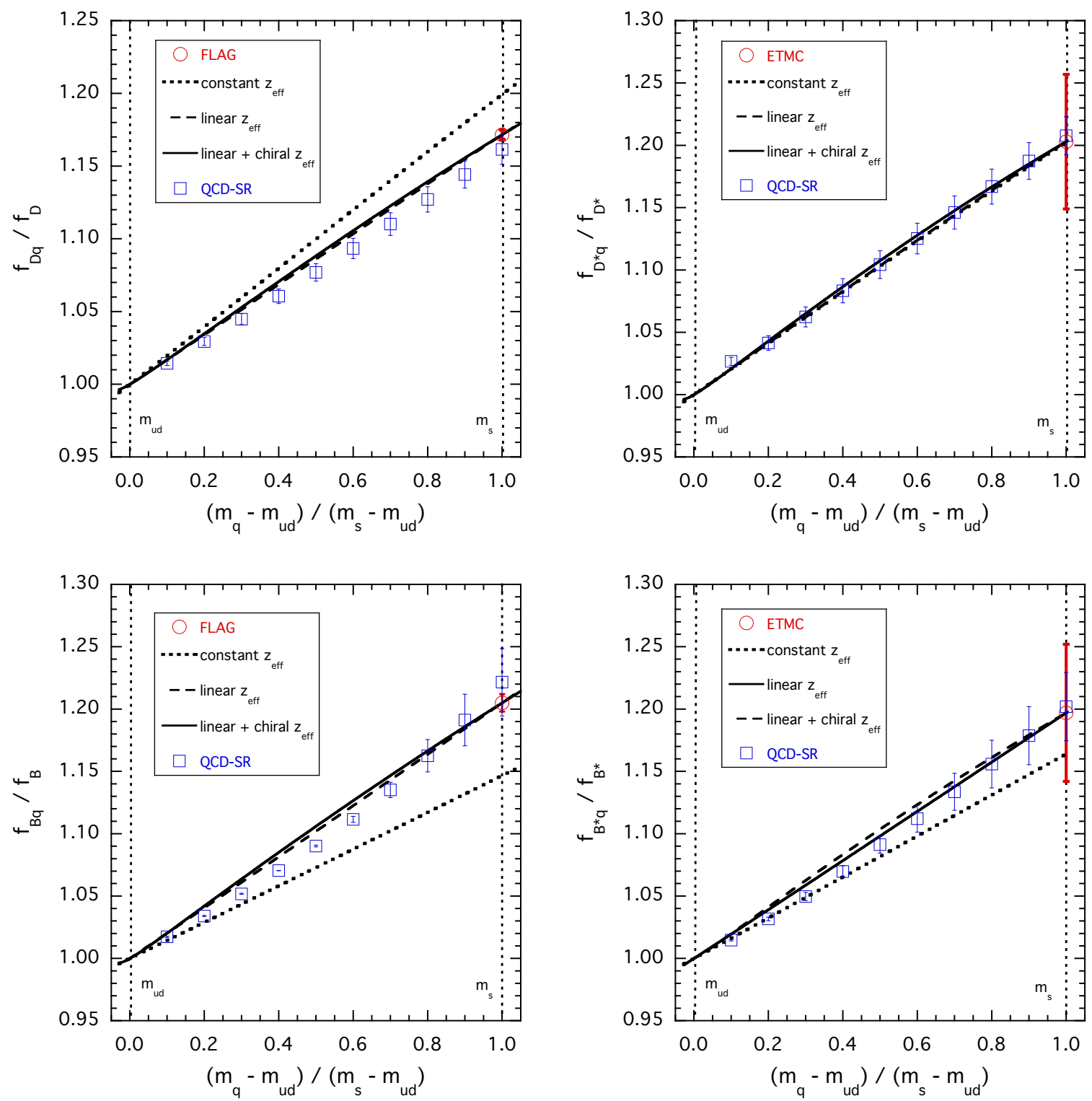

Figure 3: Decay-constant ratio $f_{H_{q}}\left(m_{q}\right) / f_{H_{q}}\left(m_{u d}\right), m_{u d} \equiv \frac{1}{2}\left(m_{u}+m_{d}\right)$, as a function of the light-quark-mass variable $\left(m_{q}-m_{u d}\right) /\left(m_{s}-m_{u d}\right)$ for three different ansätze (constant, linear, and linear plus chiral logarithms) for redefined effective threshold $z_{\text {eff }} \equiv \sqrt{s_{\text {eff }}}-m_{Q}-m_{q}$ [9], compared with the findings (squares) of Ref. [8]. 
at the light-quark masses $m_{q}=m_{d}$ and $m_{q}=m_{u}$; in that difference, all dependence on the strange sea quarks cancels, reducing greatly the uncertainties induced by neglect of $m_{\text {sea }}$ in $\rho_{2}\left(s, m_{Q}, m_{q} \mid m_{\text {sea }}\right)$ :

$$
\begin{aligned}
\delta \digamma & \equiv \digamma\left(s_{\mathrm{eff}}\left(m_{d}\right), m_{d} \mid m_{\mathrm{sea}}\right)-\digamma\left(s_{\mathrm{eff}}\left(m_{u}\right), m_{u} \mid m_{\mathrm{sea}}\right) \\
& =\digamma\left(s_{\mathrm{eff}}\left(m_{d}\right), m_{d} \mid m_{\mathrm{sea}}=0\right)-\digamma\left(s_{\mathrm{eff}}\left(m_{u}\right), m_{u} \mid m_{\mathrm{sea}}=0\right)+O\left(\frac{\alpha_{\mathrm{s}}^{2}}{\pi^{2}}\left(m_{d}-m_{u}\right)\right) .
\end{aligned}
$$

The dependence of $\digamma\left(s_{\text {eff }}\left(m_{q}, \cdot\right), m_{q} \mid m_{\text {sea }}\right)$ on $m_{q}$ originates basically in two quantities therein: The one of the spectral densities we easily extract by adapting results available in the literature [12]. The one of the effective thresholds $s_{\text {eff }}\left(m_{q}\right)=s_{0}+s_{1} m_{q}+\cdots$ we derive by allowing the light-quark mass $m_{q}$ to vary continuously between chiral limit, $m_{q}=0$, and strange-quark mass, $m_{q}=m_{s}$, defining an $H_{q}$-meson decay constant function $f_{H}\left(m_{q}\right)$ by the emerging outcomes of the local-duality QCD sum rule (1.1) for $m_{q} \in\left[0, m_{s}\right]$, and matching (cf. Fig. 3) the behaviour of $f_{H}\left(m_{q}\right)$ to lattice-QCD findings [13] for $f_{H}\left(\left(m_{u}+m_{d}\right) / 2\right)$ and $f_{H}\left(m_{s}\right)$. From the hence fully determined $m_{q}$ dependence of the QCD sum-rule outcome and numerical values of all QCD parameters in the modified minimal-subtraction renormalization scheme, we predict, for the $D, D^{*}, B$ and $B^{*}$ mesons, the decay-constant differences

$$
\begin{array}{ll}
f_{D^{ \pm}}-f_{D^{0}}=(0.96 \pm 0.09) \mathrm{MeV}, & f_{D^{* \pm}}-f_{D^{* 0}}=(1.18 \pm 0.35) \mathrm{MeV}, \\
f_{B^{0}}-f_{B^{ \pm}}=(1.01 \pm 0.10) \mathrm{MeV}, & f_{B^{* 0}}-f_{B^{* \pm}}=(0.89 \pm 0.30) \mathrm{MeV} .
\end{array}
$$

The proximity of the $f_{H}\left(m_{q}\right)$ curves resulting from constant (i.e., $m_{q}$-independent) and non-constant (i.e., $m_{q}$-dependent) parametrizations of $z_{\text {eff }} \equiv \sqrt{s_{\text {eff }}}-m_{Q}-m_{q}$ in Fig. 3 shows that roughly $70-80 \%$ of the strong isospin breaking in decay constants is due to our spectral densities' - analytically and rigorously derivable $-m_{q}$ behaviour enabling us to retain control over the accuracy of our findings.

\section{Acknowledgments}

D. M. acknowledges support by the Austrian Science Fund (FWF) under Project P 29028-N27.

\section{References}

[1] M. A. Shifman, A. I. Vainshtein, and V. I. Zakharov, Nucl. Phys. B 147 (1979) 385.

[2] W. Lucha, D. Melikhov, and S. Simula, Phys. Rev. D 76 (2007) 036002, arXiv:0705.0470 [hep-ph]; Phys. Lett. B 657 (2007) 148, arXiv:0709.1584 [hep-ph]; Phys. Atom. Nucl. 71 (2008) 1461; Phys. Lett. B 671 (2009) 445, arXiv:0810.1920 [hep-ph]; D. Melikhov, Phys. Lett. B 671 (2009) 450, arXiv:0810.4497 [hep-ph].

[3] W. Lucha, D. Melikhov, and S. Simula, Phys. Rev. D 79 (2009) 096011, arXiv:0902.4202 [hep-ph]; J. Phys. G 37 (2010) 035003, arXiv:0905.0963 [hep-ph]; Phys. Lett. B 687 (2010) 48, arXiv:0912.5017 [hep-ph]; Phys. Atom. Nucl. 73 (2010) 1770, arXiv:1003.1463 [hep-ph]; W. Lucha, D. Melikhov, H. Sazdjian, and S. Simula, Phys. Rev. D 80 (2009) 114028, arXiv:0910.3164 [hep-ph].

[4] W. Lucha, D. Melikhov, and S. Simula, J. Phys. G 38 (2011) 105002, arXiv:1008.2698 [hep-ph]; Phys. Lett. B 701 (2011) 82, arXiv:1101.5986 [hep-ph]; Phys. Rev. D 88 (2013) 056011, arXiv:1305.7099 [hep-ph]; PoS (EPS-HEP 2013) 363, arXiv:1309.5611 [hep-ph]; Phys. Lett. B 735 (2014) 12, arXiv:1404.0293 [hep-ph]; EPJ Web Conf. 80 (2014) 00043, arXiv:1407.5512 [hep-ph]. 
[5] W. Lucha, D. Melikhov, and S. Simula, EPJ Web Conf. 80 (2014) 00046, arXiv:1410.6684 [hep-ph]; arXiv:1411.3890 [hep-ph]; AIP Conf. Proc. 1701 (2016) 050007, arXiv:1411.7844 [hep-ph]; Phys. Rev. D 91 (2015) 116009, arXiv:1504.03017 [hep-ph]; PoS (EPS-HEP2015) 532, arXiv:1508.07595 [hep-ph].

[6] Particle Data Group (C. Patrignani et al.), Chin. Phys. C 40 (2016) 100001 and 2017 update.

[7] W. Lucha, D. Melikhov, and S. Simula, EPJ Web Conf. 129 (2016) 00026, arXiv:1609.02382 [hep-ph]; EPJ Web Conf. 137 (2017) 06017, arXiv:1609.09388 [hep-ph].

[8] W. Lucha, D. Melikhov, and S. Simula, Phys. Lett. B 765 (2017) 365, arXiv:1609.05050 [hep-ph].

[9] W. Lucha, D. Melikhov, and S. Simula, preprint HEPHY-PUB 982/17, arXiv:1702.07537 [hep-ph]; PoS (EPS-HEP 2017) 669, arXiv:1709.02131 [hep-ph].

[10] V. A. Nesterenko and A. V. Radyushkin, Phys. Lett. B 115 (1982) 410; 128 (1983) 439; A. P. Bakulev and A. V. Radyushkin, Phys. Lett. B 271 (1991) 223; A. V. Radyushkin, Acta Phys. Pol. B 26 (1995) 2067, arXiv:hep-ph/9511272.

[11] V. Braguta, W. Lucha, and D. Melikhov, Phys. Lett. B 661 (2008) 354, arXiv:0710.5461 [hep-ph]; I. Balakireva, W. Lucha, and D. Melikhov, Phys. Rev. D 85 (2012) 036006, arXiv:1110.6904 [hep-ph]; W. Lucha and D. Melikhov, J. Phys. G 39 (2012) 045003, arXiv:1110.2080 [hep-ph]; I. Balakireva, W. Lucha, and D. Melikhov, J. Phys. G 39 (2012) 055007, arXiv:1103.3781 [hep-ph].

[12] K. G. Chetyrkin and M. Steinhauser, Phys. Lett. B 502 (2001) 104, arXiv:hep-ph/0012002; Eur. Phys. J. C 21 (2001) 319, arXiv:hep-ph/0108017; M. Jamin and B. O. Lange, Phys. Rev. D 65 (2002) 056005, arXiv:hep-ph/0108135; P. Gelhausen, A. Khodjamirian, A. A. Pivovarov, and D. Rosenthal, Phys. Rev. D 88 (2013) 014015, arXiv:1305.5432 [hep-ph]; Phys. Rev. D 89 (2014) 099901(E); 91 (2015) 099901(E).

[13] FLAG Working Group (S. Aoki et al.), Eur. Phys. J. C 74 (2014) 2890, arXiv:1310.8555 [hep-lat]; Eur. Phys. J. C 77 (2017) 112, arXiv:1607.00299 [hep-lat]; V. Lubicz, A. Melis, and S. Simula, PoS (LATTICE2016) 291, arXiv:1610.09671 [hep-lat]. 